\title{
Nonlinear analysis of gravitational wave signals based on recurrence quantification analysis
}

\author{
Zhenyan Fan ${ }^{1}$, Qianqian Chen ${ }^{1}$, Guiqi Sun $^{1}$, Nikos Mastorakis ${ }^{2}$, and Xiaodong Zhuang ${ }^{1, *}$ \\ ${ }^{1}$ Electronics \& Information College, Qingdao University, Qingdao 266071, China \\ ${ }^{2}$ Department of Electrical Engineering and Computer Science, Hellenic Naval Academy, Piraeus, Greece
}

\begin{abstract}
Recurrence plot and recurrence quantification analysis are used to analyze different features of gravitational wave signals. Firstly, the appropriate delay time and embedding dimension are respectively estimated by methods of the C_C method. One dimension time series of gravitational wave is extended to high dimension phase space by employing phase space reconstruction for studying the movement characteristic of neighboring points in time series. Then the recurrence plots of different gravitational wave signals are implemented intuitively and qualitatively analyzing different features of gravitational wave signals. Different nonlinear characteristic parameters are calculated by recurrence quantification analysis (RQA) methods, such as recurrence rate, recurrence entropy, determinism rate and stratification, based on which the features of different gravitational wave signals can be well analyzed quantitatively.
\end{abstract}

\section{Introduction}

Gravitational Wave is proposed by Einstein in General Relativity (in 1916) and it is perturbation of cosmological space-time when objects accelerated movement. Gravitational Wave is the ripple in time and space, and the process of black hole collisions and neutron star collisions, the causes of supernova explosions, and how the Big Bang occurred can be observed through Gravitational Wave (GW) [1].

In 2016, Addesso P and Longo $\mathrm{M}$ discussed the use of sparse representations for Gravitational Wave data analysis. Gravitational wave chirps and bursts, as well as instrumental glitches, share the common feature of being represented by sparse Time-Frequency (TF) objects, including localized 1D-ridges, and 2D-blobs. It is then desirable to obtain TF representations of multicomponent waveforms that comply with the conflicting requirements of being free from inter-modulation artifacts, and offering as much TF resolution as possible. The proposed sparsity-promoting algorithms yield an excellent trade-off, and emerge as natural candidates to complement the existing methods for GW data analysis [2]. In 2017, Duong-Hung Pham and Sylvain Meignen put forward a generalization of the short-time Fourierbased synchro-squeezing transform using a new local estimate of instantaneous frequency. Such a technique enables not only to achieve a highly concentrated timefrequency representation for a wide variety of amplitudeand frequency modulated multi-component signals but also to reconstruct their modes with a high accuracy. They applied this method to gravitational-wave signals which was generated by the coalescence of two stellarmass black holes and found the efficiency of this new approach [3]. In 2018, Cao Zhoujian discussed the deep learning method for gravitational wave data processing by using simulation data. The discussion included the aspects of network structure, training data preparation, training optimization, generalization ability of signal recognition, representation of data character and response to feature data occlusion, which further improved the detection ability of gravitational waves [4]. This paper adopts recurrence plot and recurrence quantification analysis method to analyze the gravitational wave and gets some preliminary conclusions.

\section{Recurrence Plot and Recurrence Quantification Analysis}

\subsection{Recurrence plot}

It is a pictorial representation (or a graph) of a square matrix [5], in which the matrix elements correspond to those times at which a state of a dynamical system recurs [6]. This method provides a way to think about the periodic nature of a trajectory through a phase space, which is formed by the delayed vectors of dimension $d$. This state trajectory within the d-dimensional phase space is represented on a 2-D squared matrix with black and white dots representing ones and zeros. Both axes are time axes. Between two different time instances $i$ and $j$, the change of state of the particular dynamic process is imprinted in the 2-D plot. Using the Takens' theorem, a phase space trajectory can be obtained from a single scalar time series quantities. This 2-D plot called

\footnotetext{
* Corresponding author: xdzhuang@qdu.edu.cn
} 
Recurrence Plot $(R P)$ can resolve several quarries. $R P$ can be mathematically expressed as [7]

$$
R_{i j}=H\left(r-\left\|X_{i}-X_{j}\right\|\right), x_{i} \in R^{m}, i, j=1, \ldots, N
$$

Where $R_{i j}$ is the $R P, N$ is the number of considered states $X_{i}, r$ is a threshold distance, and $H\left(^{*}\right)$ is the Heaviside step function. $x(i)$ is the time series. The phase space vector $X_{i}$ has been reconstructed from this onedimensional data series with the help of Taken's time delay method as $\left\lfloor X_{i}=\left(x_{i}, x_{i+\tau}, \ldots, x_{i+(m-1) \tau}\right)\right\rfloor$ where embedding dimension is $m$ and time delay is $\tau[8]$.

When the value of $R_{i j}$ is 1 , there is a black dot in the RP. But when the value of $R_{i j}$ is 0 , there is a white dot in the RP. Recurrence plots depict a recursive relationship between states through a two-dimensional matrix of black and white dots [9].

\subsection{Recurrence Quantification Analysis}

Though the pattern texture of the RP indicates the nature of the time series, it's tedious to interpret by visual inspection when the texture is complex. The recurrence quantification analysis (RQA) overcomes this problem by estimating certain indices like $R R, D E T, L A M$ and $E N T R$, which signify various features of the time series [10].

Recurrence Rate $(R R)$ is the first recurrence variable, which is the relative quantity of recurrent points in the recurrence plot. And a higher $R R$ indicates a stronger cyclical embedding process. The expression for $R R$ is as follows [11]:

$$
R R=\frac{1}{N^{2}} \sum_{i, j=1}^{N} R_{i j}
$$

$D E T$ is the second recurrence variable, which measures the ratio of recurrence points forming diagonal structures to all recurrence points. The determinism describes the degree of the trajectory periodic recurrence. For a periodic signal, $D E T$ is close to $100 \%$, for a stochastic signal it tends toward $0 \%$ value, whereas for a chaotic signal its value varies in between that of periodic and stochastic signals. The expression for DET is as follows [12]:

$$
D E T=\frac{\sum_{l=l_{\min }}^{N} l P(l)}{\sum_{i, j=1}^{N} R_{i j}}
$$

Where $P(l)$ is the diagonal distribution probability, $l_{\min }$ is the minimum diagonal length, default is 2 .

The third recurrence variable is Entropy (ENTR), which indicates the amount of dynamic information or the stochastic degree. The expression for ENTR is as follows [8]:

$$
E N T R=-\sum_{l=l_{\min }}^{N} p(l) \ln p(l)
$$

Where $P(l)$ is diagonal distribution probability density (the length is $l$ ).

A low entropy value indicates that less information is sufficient to recognize the system, whereas high entropy indicates that much information are required to do the same. For periodic behavior, the corresponding entropy is high, whereas for chaotic behavior the entropy is low [8].

$L A M$ is the forth recurrence variable, which is the radio of the number of recurrence points in vertical line and the number of recurrence points in all vertical structure. The expression for $L A M$ is as follows [13]:

$$
L A M=\frac{\sum_{v=v_{\min }}^{N} v P(v)}{\sum_{i, j}^{N} R_{i j}}
$$

Where $P(v)$ is the frequency distribution of vertical or horizontal lines(length is $v$ ), $v_{\min }$ is the minimum analysis length, default is 2 .

The $L A M$ describes the dynamic characteristics of the system's relative, and if the $L A M$ is 0 , it indicates that the relative change of the signal is fast.

\section{Gravitational Wave Signals of Recurrence Quantification Analysis}

\subsection{Recurrence Plot}

The experiments adopt gravitational wave data from Events of LIGO Open Science Center and select strain data (H1 and L1) after noise subtraction of three events, including GW170608, GW170814 and GW170817. The sample frequency of gravitational wave data is $4096 \mathrm{~Hz}$. And 3000 data are taken from the three sets of data for constructing recurrence plot. Firstly phase space is reconstructed for gravitational wave signals by using $\mathrm{C} \_\mathrm{C}$ method and embedding dimension $\mathrm{m}$ and delay time $\tau$ are calculated by this method [14]. Then a suitable threshold $r$ is selected to make recurrence plots. The recurrence plots of gravitational wave signals are shown from Fig. 1 to Fig. 6.

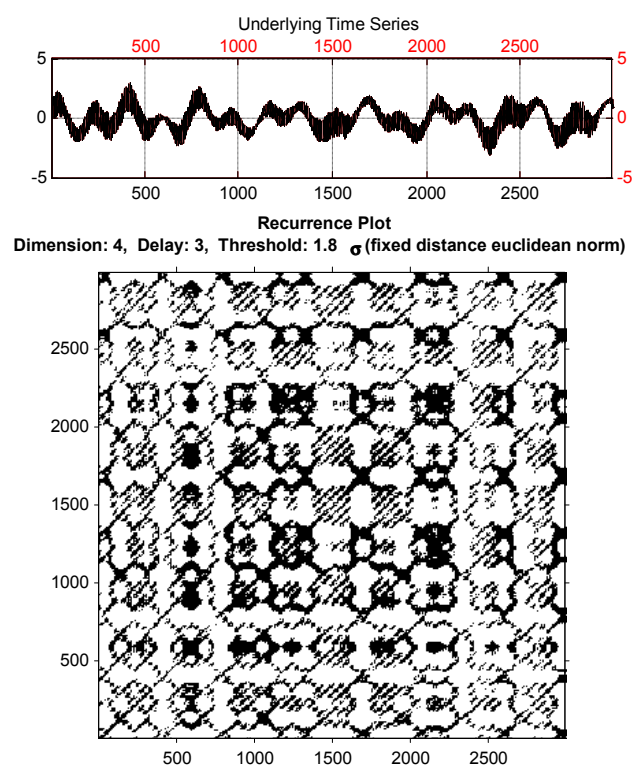

Fig. 1. Gravitation-wave signals from GW170608 H1 


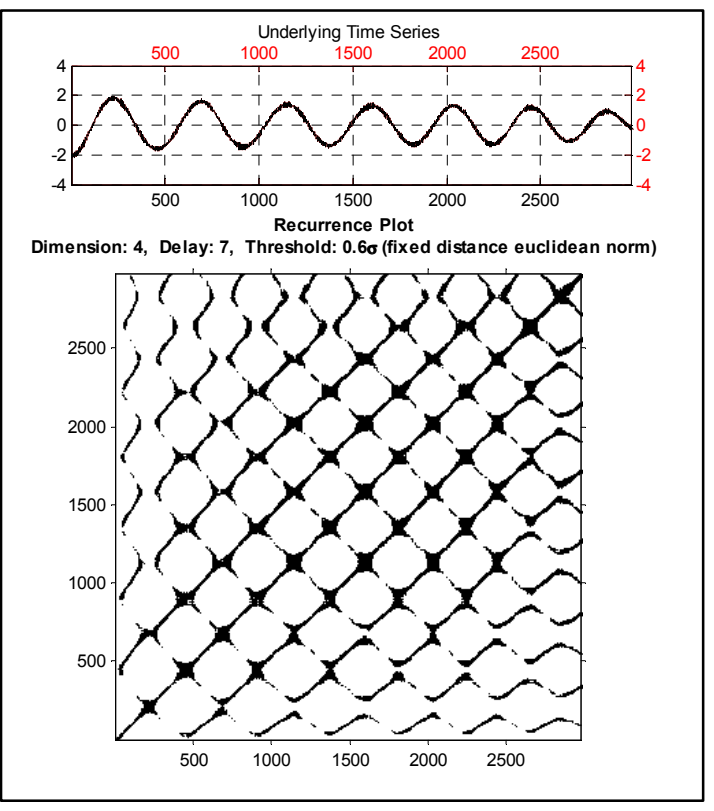

Fig. 2. Gravitation-wave signals from GW170608 L1

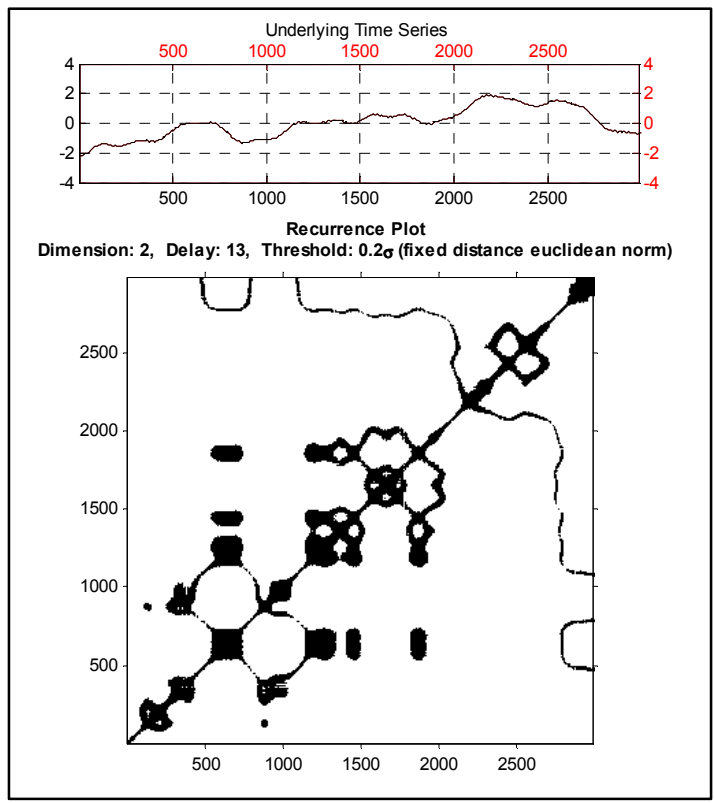

Fig. 3. Gravitation-wave signals from GW170814 H1

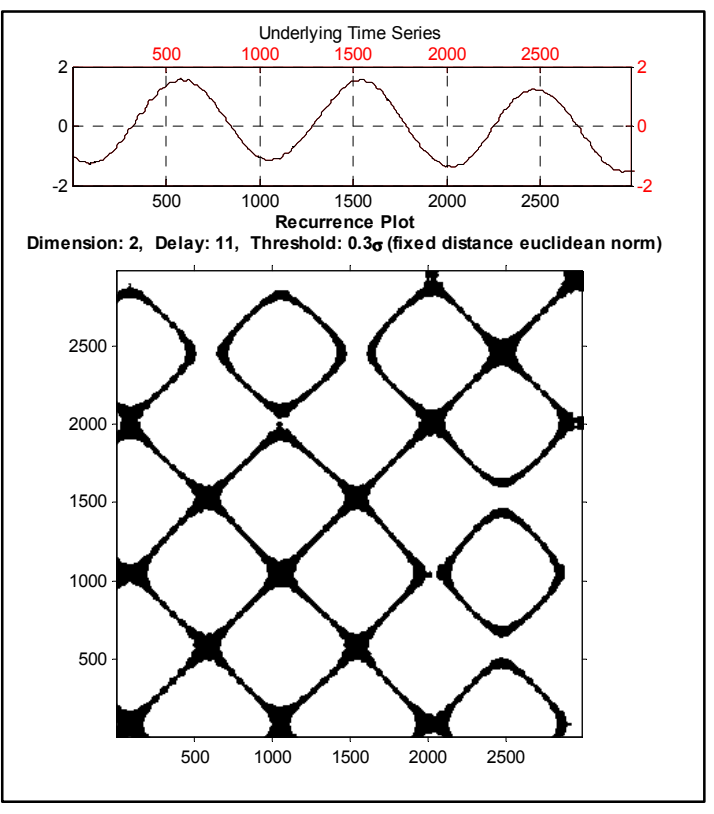

Fig. 4. Gravitation-wave signals from GW170814 L1

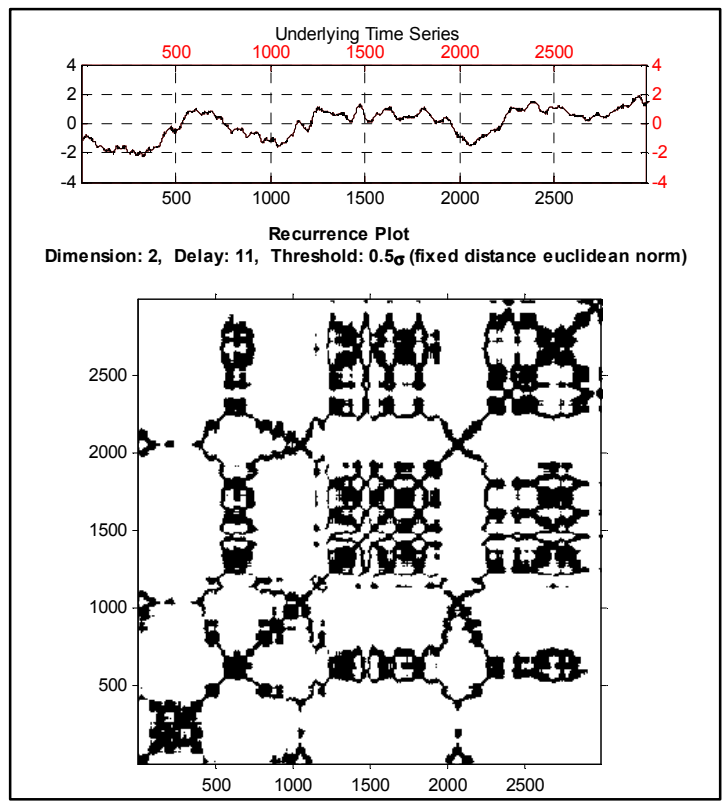

Fig. 5. Gravitation-wave signals from GW170817 H1 


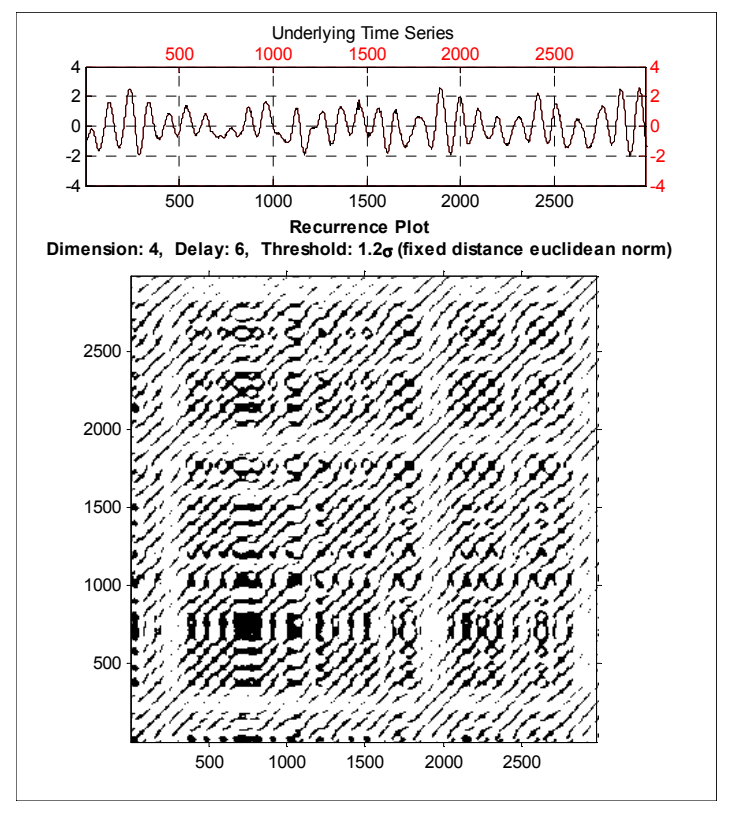

Fig. 6. Gravitation-wave signals from GW170817 L1

From Fig. 1 to Fig. 6, it can be seen that there are different features in different recurrence plots, but all recurrence plots have a main diagonal and the recurrence plots are symmetry. The recurrence plots of the stationary signal are uniformly distributed, and there is no straight line parallel to the main diagonal. Provided that the line exists, it means that there is a deterministic component in the signal. As the figures show, there are more or less straight lines parallel to the main diagonal in figure 1 , figure 2 , figure 4 and figure 6 , so there is a deterministic component in these gravitational wave signals. There are mutant structure and blank areas in Fig. 3 and Fig. 5, which indicates partial determinacy and the dramatic changes in the system.

\subsection{Recurrence Quantification Analysis}

From the recurrence plots of gravitational wave signals above, it can be obviously seen that different features of gravitational wave signals only can be observed from the images qualitatively. Therefore, this paper adopts the recurrence quantification analysis method to process gravitation wave signals by using CRP TOOL [15]. Four feature parameters, including $R R, D E T, E N T R$ and $L A M$, are selected as the main parameters. The quantification analysis results are shown in Table 1 .

From Table 1, it can be seen that the RR of GW170608_H1 is the largest but the RR of GW170814_H1 is the smallest. A higher RR indicates a stronger periodicity embedding process, so the periodicity embedding process of GW170608_H1 is the strongest. It indicates strong regularity of signals that the value of DET is $100 \%$. The DET of GW170608_H1 is smaller than that of the other five signals, so regularity of GW170608_H1 is weaker than others. The value of ENTR indicates the complexity of the deterministic structure of recurrence plot. The larger the ENTR is, the more complicated the signal is. The ENTR of GW170814_L1 is largest, so the GW170814_L1 is most complicated and has strongest chaos. LAM describes the dynamic characteristics of the system's relative change, and if the value of LAM is 0 , signals change relatively faster. The LAM of GW170608 H1 is the smallest, which indicates GW170608_H1 changes faster than the other five signals.

Table 1. The quantification analysis results

\begin{tabular}{|c|c|c|c|c|}
\hline Signals & $R R$ & $D E T$ & ENTR & LAM \\
\hline GW170608_H1 & 0.2939 & 0.8771 & 1.9950 & 0.8759 \\
\hline GW170608_L1 & 0.1325 & 0.9668 & 2.4127 & 0.9715 \\
\hline GW170814_H1 & 0.0970 & 0.9993 & 4.5830 & 0.9995 \\
\hline GW170814_L1 & 0.1348 & 0.9999 & 4.6079 & 1.0000 \\
\hline GW170817_H1 & 0.2140 & 0.9908 & 3.1651 & 0.9934 \\
\hline GW170817_L1 & 0.2103 & 0.9972 & 4.0780 & 0.9986 \\
\hline
\end{tabular}

\section{Conclusion}

In this paper, the recurrence characteristics of different gravitational wave signals is studied by adopting recurrence plot and recurrence quantification analysis method. Unidentifiable features in one-dimensional time series are extended to high-dimensional phase space based on phase space reconstruction method. It can be founded that recurrence quantification analysis method can analyze different gravitational wave signals quantitatively by calculating nonlinear features, such as $R R, D E T, E N T R$ and LAM. In future work, more nonlinear and chaotic features of gravitational wave signals will be studied.

\section{References}

1. Z. Du, Z.X. Lin, Y.Q. Gu, Computer Science, 44, 26-32 (2017)

2. P. Addesso, M. Longo, S. Marano, , Sonar and Remote Sensing. IEEE, 154-158 (2016)

3. D.H. Pham, S. Meignen, IEEE Transactions on Signal Processing, 65, 3168 - 3178 (2017)

4. Z. Cao, Journal of Henan Normal University (Natural Science Edition ), 46, 26-39 (2018)

5. N. Marwan, M.C. Romano, M. Thiel, Physics Reports, 438, 237-329 (2007)

6. N. Marwan, J. Kurths, Mathematical Physics Research at the Cutting Edge, 101-139 (2004)

7. S. Mukherjee, R. Ray, R. Samanta, M. H. Khondekar, G. Sanyal, Chaos Solitons \& Fractals, 96, 23-29 (2017)

8. J. Yu, J. Cao, W. Wang, W. Liao, Journal of Xi'an Jiaotong University, 51, 47-52 (2017)

9. R. Guo, Y. Wang, J. Yan, H. Yan, Y. Yang, Journal of Integrative Medicine, 09, 1226-1233 (2011)

10. N. Marwan, N. Wessel, U. Meyerfeldt, A. Schirdewan, J. Kurths, Phys Rev E, 66, 1-8 (2002) 
11. A.K. Chaou, A. Mekhaldi, M. Teguar, IEEE Transactions on Dielectrics \& Electrical Insulation, 22, 3376-3384 (2016)

12. C. Xia, R. Liu, Y. Wang, International Conference on Biomedical Engineering and Informatics. IEEE, 1-5 (2009)

13. C. Feng, G. Ji, J. Cheng, Periodical of Ocean University of China, 44, 106-113 (2014)

14. L. Tang, J. Liang, International Conference on Intelligent Control and Information Processing. IEEE, 1, 438-441 (2011)

15. Cross recurrence plot toolbox for Matlab, http://tocsy.agnld.uni-postdam.de., (2006) 